\title{
Constructing Space Time from the String Worldsheet
}

\author{
Mark D. Roberts \\ Flat 44, The Cloisters, 83 London Road, Guildford, GU1 1FY, UK \\ Email: robemark@gmail.com
}

\begin{abstract}
In a certain sense Riemannian geometry can be thought of as geometry built up from the Finslerian properties of point particles. The string and membrane generalization of this to a geometry similar but not the same as Finslerian geometry is investigated. Solely classical arguments suggest a physical interpretation in which microscopic strings are directly related to macroscopic geometry; alternatively the resulting geometry can be interpreted as that describing microscopic spacetime. The construction presented can be thought of as providing a mechanism for oxidization.
\end{abstract}

Keywords: Dimension, dimensional increase, finsler-like geometry, membrane.

\section{Introduction}

A problem with string theory is the nature of the relationship between microscopic strings and macroscopic spacetime. A property of string theory is that, in the limit that the string becomes a point, a corresponding field theory is recovered. General relativity is based upon geometry defined at points: from a Finslerian point of view the Riemmanian geometry of general relativity is just a macroscopic geometry constructed from the point particles' Lagrangian. This leads to the question of what sort of geometry corresponds to the string and membrane Lagrangians. This is the problem looked at here. An alternative way of looking at a physical motivation for the construction presented here is given two dimensional physics where how and why do higher dimensions come about, in particular $d=4$ spacetime from $d=2$ strings. One builds up higher dimensions on point particles for general relativity and end up with something similar to Finsler geometry, build up from the string worldsheet the result is more complex. This is the only paper that attempts to mathematically model any mechanism for increasing the number of dimensions from two dimensions: that there is a higher dimensional ambient space is usually just assumed by fiat. Throughout only classical cases where $\hbar$ is absent are looked at.

Geometry based upon area, although not world sheet area, has been studied by Cartan [7], see also Akivis and Rosenfeld [2], Brickell [6] and Vacaru [17]; Brickell considers dependence on position and area. The difference of these approaches to the present one is that dependence on velocities is also needed. In modern canonical gravity objects which are not point-like are sometimes studied, see for example Abdalla, Castello-Branco and Lima-Santos [1]. Punzi, Schuller and Wohlfarth [13] have studied motion in Finsler spaces. Spacetime as an emergent structure has been studied by Barcelo, Liberati and Visser [3], Bekenstein [5] and Pavsic [12]. An introduction to Finsler geometry has given by Chern [8]; textbooks on Finslerian geometry include Bejancu [4], Matsumato [11] and Rund [16], here Rund Chap.1 is followed wherever possible. The string worldsheet is $d=2$ and spacetime is $d=4$, to get to higher dimensions one 'oxidizes' see for example [10]; so the construction presented here can be thought of as providing a mechanism for oxidization. The terminology used here is that $\hat{g}$ is the hat metric and $g$ is the indicial metric used for simple raising and lowering of indices. The systems that Rund [16] considers have Hamiltonian normalized to one, as here systems which are weakly zero are often used, Rund's Hamiltonian approach is not looked at. The connection and curvature can be defined several ways, here we use the familiar Christoffel connection and Riemann curvature except that now the metric can be velocity dependent.

$$
\left[\begin{array}{ll}
\text { microscopic } & \text { macroscopic } \\
\text { point particle } & \text { finsler geometry } \\
\text { string } & \text { new geometry }
\end{array}\right]
$$




\section{Finsler Geometry}

Following Rund [16] pages 1-23 assume that a given space has points, curves, and velocities

$$
x^{i}, \quad x^{i}(t), \quad \dot{x}^{i} \equiv \frac{\mathrm{d} x^{i}(t)}{\mathrm{d} t}, \quad \sum_{i}\left(\dot{x}^{i}\right)^{2} \neq 0 .
$$

The distance between two close points $A\left(x^{i}\right)$ and $B\left(x^{i}+\mathrm{d} x^{i}\right)$ is given by

$$
\mathrm{d} s=F\left(x^{i}, \mathrm{~d} x^{j}\right) .
$$

Introduce the notation

$$
F_{\dot{x}^{i}}(x, \dot{x}) \equiv \frac{\partial}{\partial \dot{x}^{i}} F(x, \dot{x}) .
$$

The function $F\left(x^{i}, \dot{x}^{i}\right)$ is positively homogeneous of degree one in the velocities $\dot{x}^{i}$

$$
F\left(x^{i}, k \dot{x}^{i}\right)=k F\left(x^{i}, \dot{x}^{i}\right), \quad k>0 .
$$

Euler's theorem on homogeneous functions can be expressed as

$$
F_{\dot{x}^{i}}(x, \dot{x}) \dot{x}^{i}=F(x, \dot{x}), \quad F_{\dot{x}^{i} \dot{x}^{j}}(x, \dot{x}) \dot{x}^{i}=0, \quad \operatorname{det}\left|F_{\dot{x}^{i} \dot{x}^{j}}\right|=0 .
$$

Using the notation

$$
F \equiv F(x, \dot{x}), \quad F_{\dot{x}^{i}}^{2} \equiv\left(F^{2}\right)_{\dot{x}^{i}}=\left(F(x, \dot{x})^{2}\right)_{\dot{x}^{i}},
$$

the chain rule is

$$
\frac{1}{2} F_{\dot{x}^{i} \dot{x}^{j}}^{2}=F_{\dot{x}^{i}} F_{\dot{x}^{j}}+F F_{\dot{x}^{i} \dot{x}^{j}},
$$

multipling by $\dot{x}^{i} \dot{x}^{j}$ and applying Euler's theorem (Eq. (6)) gives the second order equality

$$
\frac{1}{2} F_{\dot{x}^{i} \dot{x}^{j}}^{2}(x, \dot{x}) \dot{x}^{i} \dot{x}^{j}=F^{2}(x, \dot{x}) .
$$

One can define a hat metric

$$
\hat{g}_{i j}(x, \dot{x}) \equiv \frac{1}{2} F_{\dot{x}^{i} \dot{x}^{j}}^{2}(x, \dot{x}),
$$

then using the second order equality (Eq. (9)) gives the hat metric Finsler function relationship

$$
\dot{x}^{i} \dot{x}^{j} \hat{g}_{i j}(x, \dot{x})=F^{2}(x, \dot{x}),
$$

comparing with Eq. (3) the familar expression for infinitesimal distance is recovered. Rund [16] goes on to discuss Hamiltonian systems.

\section{The Point Particle}

For the point particle the non linear or square root form of the Lagrangian is equal to the length

$$
\mathcal{L}=F=-m \ell \equiv-m \sqrt{-\dot{x}^{2}}
$$

This Lagrangian obeys the homogeneity relationship (Eq. (5)) as

$$
F\left(x^{i}, k \dot{x}^{i}\right)=-m \sqrt{-k \dot{x}^{a} \cdot k \dot{x}^{a}}=-m k \ell=k F .
$$

Euler's homogeneous equations (Eq. (6)) become

$$
p^{i} \equiv F_{\dot{x}_{i}}=\frac{m \dot{x}_{i}}{\ell}, \quad \dot{x}^{i} F_{\dot{x}_{i}}=-m \ell, \quad w^{i j} \equiv F_{\dot{x}^{i} \dot{x}^{j}}=\frac{m}{\ell} h_{i j}, \quad \dot{x}^{i} F_{\dot{x}_{i} \dot{x}_{j}}=0,
$$


where the projection operator

$$
h_{i j} \equiv g_{i j}-\frac{\dot{x}_{i} \dot{x}_{j}}{\dot{x}^{2}}
$$

is the familar one from general relativity, and $p^{i}$ and $w^{i j}$ and the momentum and Hessain, compare Eq. (12) [14]. The second order relationship (Eq. (9)) becomes

$$
F_{\dot{x}^{i}}^{2}=-2 m^{2} \dot{x}^{i}, \quad F_{\dot{x}^{i} \dot{x}^{j}}^{2}=-2 m^{2} g_{i j}, \quad \frac{1}{2} F_{\dot{x}^{i} \dot{x}^{j}}^{2} \dot{x}^{j}=F F_{\dot{x}_{i}}, \quad \frac{1}{2} F_{\dot{x}^{i} \dot{x}^{j}}^{2} \dot{x}^{i} \dot{x}^{j}=F^{2},
$$

From the second equation (Eq. (16)) the hat metric Finsler function relationship is given by

$$
\hat{g}_{i j} \equiv-\frac{1}{2 m} F_{\dot{x}_{i} \dot{x}_{j}}^{2}
$$

the minus sign coming about because of Lorentz signature, for a positive definite signature there would be a plus sign. In terms of the momentum and Hessian (Eq. (14)), Eq. (17) is

$$
\hat{g}_{i j}=-\frac{1}{m} p_{i} p_{j}+\ell w_{i j}
$$

and the hat metric Finsler function relationship Eq. (11) becomes

$$
\dot{x}^{i} \dot{x}^{j} \hat{g}_{i j}=-\frac{1}{m} F^{2} \text {. }
$$

One difference is that from Eq. (6)

$$
\operatorname{det}\left|F_{\dot{x}^{i} \dot{x}^{j}}\right|=\frac{m}{\ell} \operatorname{det}|-h|
$$

It is apparent that $\hat{g}=g$ so that the metric and geometry are the same as that of general relativity.

\section{The String}

For simplicity a specific string parameterization is looked at now: a parameterization invariant approach is considered in the next section Sect. 5. The string the square root or non linear Lagrangian is equal to the area

$$
\mathcal{L}=F=-\frac{\mathcal{A}}{2 \pi \alpha^{\prime}}, \quad \mathcal{A} \equiv \sqrt{\left(\dot{x} \cdot x^{\prime}\right)^{2}-\dot{x}^{2} x^{\prime 2}},
$$

where $\dot{x}=\partial_{\tau} x$ and $x^{\prime}=\partial_{\sigma} x, \tau$ is the proper time of the string and $\sigma$ is the spanning parameter. In the velocity only approach $F=F\left(x^{i}, \dot{x}^{i}\right)$ in particular $F$ is not a function of $x^{\prime}$. For the string use $F(x, \dot{x})$ as before and the $x^{\prime}$ come out as new terms, there are other possibilities that are discussed under membranes Eq. (43). The Lagrangian Eq. (21) obeys the homogeneity relationship (Eq. (5)) as

$$
F\left(x^{i}, k \dot{x}^{i}\right)=-\frac{1}{2 \pi \alpha^{\prime}} \sqrt{k^{2}\left(\dot{x} \cdot x^{\prime}\right)^{2}-k \dot{x}^{a} \cdot k \dot{x}^{a} x^{\prime 2}}=-\frac{k}{2 \pi \alpha^{\prime}} \mathcal{A}=k F,
$$

Euler's homogeneous equations (Eq. (6)) become

$$
\begin{aligned}
& p^{i} \equiv F_{\dot{x}_{i}}=\frac{1}{2 \pi \alpha^{\prime} \mathcal{A}}\left(-\dot{x} \cdot x^{\prime} x_{i}^{\prime}+x^{\prime 2} \dot{x}_{i}\right), \quad \dot{x}^{i} F_{\dot{x}_{i}}=\frac{-\mathcal{A}}{2 \pi \alpha^{\prime}} \\
& w^{i j} \equiv F_{\dot{x}_{i} \dot{x}_{j}}=\frac{x^{\prime 2} h^{i j}}{2 \pi \alpha^{\prime} \mathcal{A}}, \quad \dot{x}^{i} F_{\dot{x}^{i} \dot{x}^{j}}=0
\end{aligned}
$$

where the projection tensor is

$$
h^{i j} \equiv g^{i j}+\frac{1}{\mathcal{A}^{2}}\left(\dot{x}^{2} x^{i} x^{\prime j}+x^{\prime 2} \dot{x}^{i} \dot{x}^{j}-\left(\dot{x} \cdot x^{\prime}\right)\left(\dot{x}^{i} x^{\prime j}+x^{\prime i} \dot{x}^{j}\right)\right), h=d-2,
$$


and $d$ is the dimension of the space and $h$ is the dimension of the projection. compare [14, Sect. 3.4] . The second order relationship (Eq. (9)) becomes

$$
\begin{aligned}
& F_{\dot{x}^{i}}^{2}=-\frac{\mathcal{A}}{\pi \alpha^{\prime}} p_{i}, \quad F_{\dot{x}_{i} \dot{x}_{j}}^{2}=\frac{1}{2 \pi^{2} \alpha^{\prime 2}}\left(x^{\prime i} x^{\prime j}-x^{\prime 2} g_{i j}\right), \\
& \frac{1}{2} F_{\dot{x}^{i} \dot{x}^{j}}^{2} \dot{x}_{j}=F F_{\dot{x}^{i}}, \quad \frac{1}{2} F_{\dot{x}^{i} \dot{x}^{j}}^{2} \dot{x}^{i} \dot{x}^{j}=F^{2},
\end{aligned}
$$

The hat metric $\hat{g}$ (Eq. (17)) generalizes to

$$
\hat{g}_{i j} \equiv-\frac{1}{2} F_{\dot{x}^{i} \dot{x}^{j}}^{2}=\frac{1}{4 \pi^{2} \alpha^{\prime 2}}\left(-x_{i}^{\prime} x_{j}^{\prime}+x^{\prime 2} g_{i j}\right),
$$

and in this case does not equal the indicial metric $g$. In terms of the momentum and Hessian(Sect. 4), Eq. (26) is

$$
\hat{g}_{i j}=-p_{i} p_{j}+\frac{\mathcal{A}}{2 \pi \alpha^{\prime}} w_{i j},
$$

the hat metric Finsler function relationship (Eq. (11)) becomes

$$
\dot{x}^{i} \dot{x}^{j} \hat{g}_{i j}=-\frac{\mathcal{A}^{2}}{4 \pi^{2} \alpha^{\prime 2}}=-F^{2} .
$$

In the present case equation (Eq. (26)) shows that the two metrics $g$ and $\hat{g}$ are not the same. Suppose one seeks an inverse of the metric (Eq. (27)) of the form

$$
\hat{g}^{i j}=\alpha \dot{x}^{i} \dot{x}^{j}+\beta\left(\dot{x}^{i} x^{\prime j}+x^{i} \dot{x}^{j}\right)+\gamma x^{i} x^{j}+b g^{i j},
$$

then using Eq. (26) to form the identity, dimension $d=\hat{g}^{i j} \hat{g}_{i j}$, the $\beta$ and $\gamma$ terms self-cancel and the $b$ term leads to a contradiction, leaving the $\alpha$ term

$$
\hat{g}^{i j}=-\frac{4 \pi^{2} \alpha^{\prime 2} d}{\mathcal{A}^{2}} \dot{x}^{i} \dot{x}^{j} .
$$

The inverse metric allows construction of a Christoffel symbol which is a sum of

$$
\begin{aligned}
\stackrel{1}{\Gamma_{j k}^{i}} & =\frac{\dot{x}^{i} d}{\mathcal{A}^{2}}\left(\dot{x} \cdot x^{\prime} x_{(j, k)}^{\prime}+\dot{x}^{l} x_{j}^{\prime} x_{[l, k]}^{\prime}+\dot{x}^{l} x_{k}^{\prime} x_{[l, j]}^{\prime}\right), \\
\stackrel{2}{\Gamma_{j k}^{i}} & =\frac{\dot{x}^{i} d}{2 \mathcal{A}^{2}}\left(-\dot{x}^{l} x^{\prime 2}\left\{{ }_{j l k}\right\}-2 \dot{x}_{(j} x_{, k)}^{\prime 2}+g_{j k} \dot{x}^{l} x^{\prime 2}, l\right),
\end{aligned}
$$

and from this a Riemann tensor can be constructed, but its interpretation is obscure.

\section{The Membrane}

The square root or non linear membrane action is

$$
S_{D}=k \int_{M} \mathrm{~d}^{\mathrm{p}+1} \xi \sqrt{-\gamma}, \quad \gamma_{\mathrm{ab}}=\mathrm{g}_{\mathrm{ij}} \partial_{\mathrm{a}} \mathrm{x}^{\mathrm{i}} \partial_{\mathrm{b}} \mathrm{x}^{\mathrm{j}}, \quad \sqrt{-\gamma}=\left(-\operatorname{det} \gamma_{\mathrm{ab}}\right)^{\frac{1}{2}},
$$

$a$ is an internal membrane index which generalizes the proper time $\tau$ and spanning parameter $\sigma$ of the previous section. The Finsler function is given by

$$
\mathcal{L}=F\left(x, x^{a}\right)=k \sqrt{-\gamma} .
$$

Choosing

$$
\begin{aligned}
& p=1, \quad k=-\frac{1}{2 \pi \alpha^{\prime}}, \quad a, b \cdots=\tau, \sigma, \quad \mathrm{d}^{2} \xi=\mathrm{d} \tau \mathrm{d} \sigma \\
& \gamma=\operatorname{det}\left(\gamma_{a b}\right)=-\mathcal{A}^{2}, \quad \gamma_{a b}=\left(\begin{array}{cc}
\dot{x}^{2} & \dot{x} \cdot x^{\prime} \\
x^{\prime} \cdot \dot{x} & x^{\prime 2}
\end{array}\right), \quad \gamma \gamma^{a b}=\left(\begin{array}{cc}
x^{\prime 2} & -x^{\prime} \cdot \dot{x} \\
-\dot{x} \cdot x^{\prime} & \dot{x}^{2}
\end{array}\right),
\end{aligned}
$$


the string Lagrangian (Eq. (21)) is recovered. The first fundamental form is defined by

$$
\aleph^{i j} \equiv \gamma^{a b} x_{a}^{i} x_{b}^{j}, \quad \aleph^{i k} \aleph_{. k}^{j}=\aleph^{i j}, \quad \aleph_{. k}^{k}=\gamma_{. c}^{c}=x_{. .}^{k c} x_{k c}=p+1,
$$

which allows the generalization of the projection tensors Eq. (15) and Eq. (24) to be expressed as

$$
h^{i j}=g^{i j}-\aleph^{i j}, \quad h=d-1-p .
$$

One can form the Christoffel symbol for the metric $\gamma$

$$
\left\{\begin{array}{l}
a \\
b c
\end{array}\right\}=x^{a} \cdot x_{b, c},
$$

using the last equation of Eq. (35) partial derivatives can be replaced by covariant derivatives

$$
x_{; b c}=x_{, b c}-\left\{\begin{array}{l}
e \\
b c
\end{array}\right\} x_{e}=x_{, b c}\left(1-x^{e} x_{e}\right)=-p x_{, b c},
$$

and Eq. (37) becomes

$$
\left\{\begin{array}{l}
a \\
b c
\end{array}\right\}=-\frac{1}{p} x^{a} \cdot x_{; b c},
$$

which must be torsion free. The Riemann tensor is defined by

$$
R_{b c d}^{a} \equiv\left\{\begin{array}{l}
a \\
d b
\end{array}\right\}_{, c}-\left\{\begin{array}{l}
a \\
c b
\end{array}\right\}_{, d}+\left\{\begin{array}{l}
a \\
c f
\end{array}\right\}\left\{\begin{array}{c}
f \\
d b
\end{array}\right\}-\left\{\begin{array}{c}
a \\
d f
\end{array}\right\}\left\{\begin{array}{c}
f \\
c b
\end{array}\right\},
$$

using Eq. (39) this is

$$
R_{b c d}^{a}=-\frac{2}{p}\left(x^{a} \cdot x_{;[d|b|}\right)_{; c]}+\frac{2}{p^{2}} x^{e} \cdot x_{;[c \mid b} x^{a} \cdot x_{; e \mid d]}
$$

contracting and using $0=(p+1)_{, e}=\left(x^{a} x_{a}\right)_{; e}=2 x^{a} x_{; a e}$ gives

$$
R_{b d}=-\frac{1}{p}\left(x^{a} \cdot x_{; d b}\right)_{; a}+\frac{1}{p^{2}} x^{e} \cdot x_{; a b} x^{a} \cdot x_{; e d}, \quad R=-\frac{1}{p}\left(x^{a} \cdot x_{d}^{d}\right)_{; a}+\frac{1}{p^{2}} x^{e} \cdot x_{; a b} x^{a} \cdot x_{; e}{ }^{b} .
$$

The Ricci scalar is a total derivative so that it has limited use as a classical Lagrangian. The Lagrangian obeys a generalization of the homogeneity condition Eq. (5) and Eq. (22)

$$
F\left(x^{i}, k_{A} x^{i A}\right)=k_{A} F,
$$

where $A$ is an unsummed internal index. From [15] the momentum and Hessian are

$$
p^{i a}=\frac{\partial \mathcal{L}}{\partial x_{i a}}=+k \sqrt{-\gamma} x^{i a}, \quad w^{i j a b}=\frac{\partial^{2} \mathcal{L}}{\partial x_{j b} \partial x_{i a}}=+k \sqrt{-\gamma}\left(g^{i j} \gamma^{a b}+x^{i a} x^{j b}\right),
$$

The generalization of the second order relationship (Sect. 4) is

$$
F_{x_{i a}}^{2}=2 k \sqrt{-\gamma} p^{i a}, \quad F_{x_{i a} x_{j b}}^{2}=-2 k^{2} \gamma\left(g^{i j} \gamma^{a b}+2 x^{b j} x^{a i}\right)
$$

from which the generalization of the hat metric (Eq. (26)) is

$$
\hat{g}^{i j} M^{a b} \equiv-\frac{1}{2} F_{x_{i a} x_{b j}}^{2}=k^{2} \gamma\left(g^{i j} \gamma^{a b}+2 x^{i a} x^{b j}\right)
$$

where $M$ is a matrix to be determined. There are three possible ways of removing the internal indices occuring in $M_{a b}$ : the first is to pick out a component

$$
\hat{g}_{i j} M_{\tau \tau}=k^{2} \gamma\left(g_{i j} \gamma_{\tau \tau}+2 \dot{x}_{i} \dot{x}_{j}\right)
$$

proceeding as for Eq. (29) there are more possibilities, but choosing that $\hat{g}$ has only explicit $\dot{x}$ dependence and not $x^{\prime}$ dependence

$$
\hat{g}^{i j}=\frac{d M_{\tau \tau} \dot{x}^{i} \dot{x}^{j}}{k^{2} \gamma \dot{x}^{2}\left(\gamma_{\tau \tau}+2 \dot{x}^{2}\right)},
$$


the second is to trace over the internal indices

$$
\hat{g}_{i j} M_{a}^{a}=k^{2} \gamma\left((p+1) g_{i j}+2 \aleph_{i j}\right)
$$

where the first fundamental form $\aleph$ is given by (Eq. (35)), the inverse metric is of the form

$$
\hat{g}^{i j}=\alpha g^{i j}+\beta \aleph^{i j},
$$

where $\alpha$ and $\beta$ are constrained by

$$
(d+2) \alpha+(p+3) \beta=\frac{d M_{c}^{c}}{k^{2} \gamma(p+1)}
$$

the third is to take determinants over the internal indices

$$
\hat{g}_{i j} \operatorname{det}\left(M_{a b}\right)=k^{2}(-\gamma)\left(-g_{i j}-2 \operatorname{det}_{a b}\left(x_{i a} x_{b j}\right)\right)
$$

where $\operatorname{det}_{a b}$ signifies that the determinant is taken over the internal indices $a, b$, this choice does not seem to have an explicit inverse because of the determinant. For Eq. (47) and Eq. (49) it is possible to construct Christoffel symbols similar to Sect. 4.

\section{Conclusion}

The Finsler point particle (Eq. (12)) allows construction of a hatted metric $\hat{g}$ (Eq. (17)) which has identical infinitesimal length $\mathrm{d} s^{2}=g_{i j} \dot{x}^{i} \dot{x}^{j}$ to that of Riemannian geometry, so that in this sense the point particle's metric generates Riemannian geometry. Given this identity it is natural to ask what sort of geometry is generated by string (Eq. (21)) and membrane (Eq. (32)) actions. It is found that the relationship between the hat metric $\hat{g}$ and the indicial metric $g$ given by Eq. (26) and Eq. (46) is no longer an equality as terms dependent on the internal properties of the string or membrane appear. This macroscopic dependence on microscopic internal properties might be small enough to produce realistic models but large enough to lead to new predictions. There is the possibility that this geometric picture might have a thermodynamic analogy, compare [9], in which entropy could be assigned to the string's area $\mathcal{A}$ and related to macroscopic properties. The geometry used does not involve $\hbar$ so that the relationship between microscopic and macroscopic is classical: a more usual picture would be to take it that quantum and many body properties of strings are necessary to build medium size systems, in other words that physically intermediate length scale properties are necessary. Alternatively the geometry presented here could be interpreted as that the geometry of microscopic spacetime.

Acknowledgements. I would like to thank David Bao, Howard E. Brandt and Lajos Tamassy for correspondence in september 2000, and Jürgen Jost for conversations in 2007 on geometry based on area.

\section{References}

1. E. Abdalla, K.H.C. Castello-Branco and A.Lima-Santos, Area Quantization in Quasi-Extreme Black Holes. gr-qc/0301130

2. M.A. Akivis and B.A. Rosenfeld, Editors, Elie Cartan (1869-1951), Translation of Mathematical Monographs, Vol. 123, PP. 225-226, American Mathematical Society (1993) (English translation of the Russian).

3. C. Barcelo, Stefano Liberati \& Matt Visser, Analogue Gravity. Living Reviews in Relativity 8(2005)12.

4. Aurel Bejancu, Finsler geometry and applications. Ellis Horwood, Chichester, England (1990). Math.Rev.91i:53075

5. Jacob D. Bekenstein, The relation between physical and geometrical gravity. gr-qc/9211017 Phys.Rev.D48(1993)3641-3647, Math.Rev.94g:83109.

6. F. Brickell, On the existence of metric differential geometries based on the notion of area. Proc.Cambridge Phys.Soc.46(1950)67-72, Math.Rev.003603

7. Elie Cartan, Les espaces metriques fondes sur la notion d'aire (Metric Spaces Based on the Notion of Area), Exposes de Geometrie, Vol.I, Hermann, Paris (1937) (French). 
8. Shiing-Shen Chern, Finsler geometry is just Riemannian geometry without the quadratic restriction, Notices of the AMS September (1996)959-963.

9. Theodore A. Jacobson, Thermodynamics of spacetime: The Eistein equation of state, Phys.Rev.Lett.75(1995)1260, Math.Rev.96f:83071.

10. H.Lü, S.Mukherji, C.N.Pope\& K.-W.Xu, Cosmological solutions in string theory, hep-th/9610107,

11. M. Matsumoto, Foundations of Finsler geometry and special Finsler spaces. Kaisishu: Shiguken (1986). Math.Rev.88f:53111

12. Matej Pavsic, An extra structure of spacetime: A space of points, areas and volumes. gr-qc/0611050

13. Raffaele Punzi, Frederic P. Schuller and Mattias N.R. Wohlfarth, Massive motion in area metric spacetimes, Phys.Rev.D79(2009)124025

14. Mark D. Roberts, The Rotation and Shear of a String. Class.Q.Grav.20(2003)507-519, Math.Rev.2003m:83134. hep-th/0204236.

15. Mark D. Roberts, The Relative Motion of Membranes. Central European Journal of Physics8(2010)915-919, gr-qc/0404094

16. Hanno Rund, The differential geometry of Finsler spaces. Springer Verlag, Heidelberg, 1959, Math.Rev.0105726.

17. Sergin I. Vacaru, P. Stavrinos, E. Gaburov and D. Gonta, Clifford and Riemann-Finsler Structures in Geometric Mechanics and Gravity, gr-qc/0508023, Math.Rev.2008i:53107 